\title{
Diagnostic Value of Plasma M2-Pyruvate Kinase in Egyptian Patients with Colorectal Cancer
}

\author{
Ali Ahmed Wahib ${ }^{1}$, Mohamed Safwat Seif El-Nasr ${ }^{1}$, Mahmoud Bastawy Ismail ${ }^{2}$, Diaa \\ Mohammad Eltiby $^{*}$, Sayed Abd-Elrehim Sayed ${ }^{3}$, Moataz Yousry Soliman ${ }^{1}$ \\ Departments of ${ }^{1}$ Tropical Medicine, ${ }^{2}$ Clinical Pathology and ${ }^{3}$ Pathology \\ Faculty of Medicine, Al-Azhar University, Cairo, Egypt \\ *Corresponding author: Diaa Mohammad Eltiby, E-mail: diaaeltebi@gmail.com
}

\begin{abstract}
:
Background: M2-pyruvate kinase (M2-PK) is a tumor growth key regulator and plays a crucial role in tumor metabolism and can dynamically regulate aerobic glycolsis. Notwithstanding, the colonoscopy is the gold standard for early detection of colorectal cancer (CRC), the acceptance of this invasive technique is low. Aim of the work: to assess the diagnostic value of plasma M2-PK in Egyptian patients with CRC. Methods: we measured the plasma M2-PK using Enzyme-linked immunosorbent assay in CRC patients and comparing these levels with those obtained from patients with functional bowel disorders (FBD), inflammatory bowel disease (IBD), colorectal polyp and apparently healthy individuals and to detect its sensitivity and specificity. Results: the value of plasma M2-PK level was higher in CRC patients, $15.22 \mathrm{U} / \mathrm{ml}$ than other groups. Average plasma M2-PK was 6.78, 5.28, 2.25 and $2.1 \mathrm{U} / \mathrm{ml}$ among colorectal polyp, IBD, FBD patients and normal individuals respectively. The plasma M2-PK can be used in differentiating between CRC and normal individual with $83.33 \%$ sensitivity, $100 \%$ specificity at area under the curve (AUC) 0.949 with cut-off $>6 \mathrm{U} / \mathrm{ml}$. Also, it can be used to discriminate between colorectal polyp and CRC patients at cut-off level of $>10.6 \mathrm{U} / \mathrm{mL}$ with $75.5 \%$ sensitivity and $87.5 \%$ specificity and to differentiate between FBD and organic colonic lesions at cut-off level $>3 \mathrm{U} / \mathrm{ml}$ with $81.94 \%$ sensitivity and $83.3 \%$ specificity. Conclusion: plasma M2-PK was considerably elevated in CRC patients and may be used as non-invasive biomarker for CRC. Recommendation: future researches including a large and long-term follow-up studies are recommended.
\end{abstract}

Keywords: Colorectal cancer; M2-Pyruvate kinase; Functional bowel disorders; IBD; colorectal Polyp.

\section{INTRODUCTION}

Colorectal cancer (CRC) is a major public health problem, being the third most commonly diagnosed cancer and the fourth reason of cancer death worldwide ${ }^{(\mathbf{1})}$.Most cases of CRC are preventable at early stage, and several randomized, controlled trials have shown lower mortality among patients who undergo screening than among those who do not ${ }^{(2,3)}$. Survival is markedly affected by the stage of the disease at the time of diagnosis ${ }^{(4)}$.

Regardless of considerable progress that has been made over the past years in diagnosis and management strategies of CRC, still many of the CRC patients are diagnosed in late stages and do not respond to treatment ${ }^{(5)}$, therefore studies should be done to improve our understanding of pathological process in the CRC for identifying the best diagnostic, prognostic, and predictive biomarkers supporting its role in prevention, early detection and treatment ${ }^{(6,7)}$.
In spite of the fact that, the gold standard for the early detection of CRC is colonoscopy; the acceptance of this invasive and expensive method is low even in more developed countries ${ }^{(\mathbf{8}, 9)}$. The selection of patients who should undergo colonoscopy and/or imaging procedures is one of the key points of the diagnostic process, which should avoid the abuse of invasive and costly tests as well as the underestimation of potentially harmful diseases. The investigation and procedures to differentiate organic from functional bowel disorders represents a considerable burden both for patients and community health service ${ }^{(\mathbf{1 0})}$.

Premalignant conditions of CRC as chronic inflammatory bowel disease (IBD) and colorectal polyp are preventable. In all such cases, recognition of the disease at an early stage is essential to devise suitable preventive cancer strategies ${ }^{(\mathbf{1 1})}$. Therefore, to develop an alternative modality based on blood biomarkers 
as the first line screening test is an alternative ideal way for the early detection of CRC ${ }^{(12)}$.

There are four pyruvate kinase isoforms presenting in human. The M1 isoform is presented in most adult and differentiated tissues; $\mathrm{L}$ and $\mathrm{R}$ isoforms are expressed in liver and red blood cells; the dimeric form of the M2 isoform is a splice variant of M1 expressed in cancer cells and undifferentiated tissues ${ }^{(13)}$. M2-pyruvate kinase (M2-PK) play an important role in tumor metabolism and researches revealed that M2-PK may react as protein kinase and co-activator of transcription factors ${ }^{(14)}$. It is a tumor growth key regulator and can dynamically regulate glycolsis energy production and synthetic processes ${ }^{(2)}$. M2-PK in CRC patients was about 4 times higher than normal individuals ${ }^{(13)}$. Plasma M2-PK could be helpful in discriminating malignant and benign colorectal lesions and may yield insight in terms of survival ${ }^{(8)}$.

\section{AIM OF THE WORK:}

The aim of the study is to determine the diagnostic value of plasma M2-PK in Egyptian patients with CRC.

\section{PATIENTS AND METHODS}

The study was conducted on 120 persons who fulfilling the designed inclusion criteria. The study was carried out in Tropical Medicine department, Al-Azhar University Hospitals (Al-Hussein and Sayed Galal Hospitals) during the period from June 2015 to June 2018.

\section{Inclusion criteria:}

- Age $\geq 18$ years.

- Patient with lower GIT symptoms and have an indication for lower endoscoy including chronic constipation, chronic diarrhea, changing in bowel habits and bleeding per rectum and other relevant alarming symptoms and signs e.g. significant unexplained weight loss, unexplained anemia, patients with remote metastases proved to be adenocarcinoma and were suspected to have CRC and patients underwent screening for CRC.

- The inclusion criteria for FBD group: confirmed diagnosis of FBD after full work up (Endoscopies, histopathology, etc).

- The inclusion criteria for the IBD patients: confirmed diagnosis of IBD was ascertained using conventional clinical, endoscopic, radiological and histopathological criteria.
-The inclusion criteria for colorectal polyp group: confirmed diagnosis by Endoscopies and histopathology other than polyp of IBD and CRC.

- The inclusion criteria for CRC group: confirmed diagnosis by Endoscopies and histopathology.

\section{Exclusion criteria:}

囚atients who refused to participate in the study.

囚 Patients who previously underwent surgery for CRC or received chemotherapy for CRC.

$\otimes$ Patients having cancer at any other site.

囚 Sepsis.

All the studied patients were subjected to: Full history taking, thorough clinical examination, imaging, colonoscopy and biopsies and routine laboratory investigations and specific tumor marker; Carcinoembryonic antigen (CEA) and plasma M2-PK.

\section{Measurement of plasma M2-PK:}

Blood was drawn from each patient into EDTA containing plasma tubes, centrifuged at the speed of 2000-3000 rpm for 20-min. Supernatant was removed, if precipitation appeared, centrifuged again. All icteric or hemolytic blood samples discarded. Plasma samples were stored at $-80^{\circ} \mathrm{C}$ until the assay was performed. Our assay carefully followed the instructions of the test kit.

The study was done according to the Ethical Board of Al-Azhar University. All subjects involved in the current study were informed about the nature and details of the work and a written consent was obtained for each participant.

One hundred twenty individuals were involved in this study and classified into five groups:

Group I: Including 24 healthy persons.

Group II: Including 24 patients with FBD (Irritable bowel syndrome: 22; functional diarrhea: 1 and functional constipation: 1).

Group III: Including 24 patients with IBD (Ulcerative colitis (UC): 23 and Crhon's Disease (CD): 1).

Group IV: Including 24 patients with colorectal polyp (adenomatous polyp: 21 ; nonadenomatous polyp: 3 ).

Group V: Including 24 patients with CRC. Statistical analysis:

Data were collected, revised, coded and entered to the Statistical Package for Social Science (IBM SPSS) version (23) for statistical analysis. Quantitative data were expressed as 
mean \pm standard deviation (SD). Qualitative data were expressed as frequency and percentage. The comparison between groups regarding qualitative data was done by using Chi-square test. The comparison between more than two independent groups with quantitative data and parametric distribution was done by using One Way ANOVA test with post hoc analysis. Also Receiver operating characteristic curve (ROC) were used to assess the best cutoff point with sensitivity, specificity, positive and negative predictive value and area under curve (AUC). The confidence interval was set to $95 \%$ and the margin of error accepted was set to $5 \%$. So, the p-value was considered significant at the level of $<0.05$ and p-value $<0.001$ was considered as highly significant.

\section{RESULTS}

Table 1 shows the demographic features of the study population. The age of FBD group was $41.80 \pm 10.82$, in IBD patient's $34.54 \pm$ 11.43 , in colorectal polyp patient $45.08 \pm 11.80$ in and CRC patients, $50.38 \pm 11.26$ years. There was no statistical significant difference between studied groups as regard gender. However, in IBD group females $(58.3 \%$ ) are more common than males while males are more common in colorectal polyp and cancer groups $62.5 \%$ and $58.3 \%$ respectively. The main presentations in our CRC patients were bleeding per rectum experienced by (33.3\%), followed by change of bowel habits about $(29.2 \%)$ then weight loss (20.8\%).

Regarding ESR, there was a significant difference between normal and FBD groups and patients with organic colonic lesions (IBD, colorectal polyp \& cancer). Also, CRP show a significant difference between the studied groups mainly elevated in IBD group as shown in table 2. According to the anatomic site of CRC the current study showed that the rectal cancer $(37.5 \%)$ was more common than proximal $(33.33 \%)$ and distal colon cancer (29.17\%). The present study showed that conventional adenocarcinoma (87.5\%) was more common than mucinous adenocarcinoma $(12.5 \%)$. Based on histologic differentiation the current study showed that the most frequent grading of the tumor was (66.7\%) moderately differentiated, followed by poorly differentiated $(20 \%)$ then well differentiated (12.5\%). Lymph nodes involvement in CRC patient was $58.3 \%$ while, evidence of distant metastasis was $16.7 \%$. Most of the patient presented at stage III (37.5\%) and stage II (33.3\%) followed by stage IV (20.8\%) and only $8.3 \%$ of patients presented at stage I.

Table 2 shows average of tumor marker of the studied groups. The mean value of CEA in normal and benign groups was nearly below 5 $\mathrm{ng} / \mathrm{mL}$ while, in CRC group its mean was 88.4 $\mathrm{ng} / \mathrm{ml}$ and median53ng/ml. CEA was higher in CRC group than other groups, with low sensitivity (66.67\%) at AUC 0.711 when compared to normal population (table 3 ).

The value of plasma M2-PK level was higher in CRC patients $15.22 \pm 8 \mathrm{U} / \mathrm{ml}$ than other groups (p-value $<0.001)$. Average plasma M2PK was $6.78 \pm 3.48,5.28 \pm 3.33,2.25 \pm$ and $2.1 \pm 1.75 \mathrm{U} / \mathrm{ml}$ among colorectal polyp, IBD, FBD patients and normal individuals respectively (table 2 ). The value of plasma M2PK level was higher in active UC 8.2 \pm 4.7 compared to inactive UC patients $3.64 \pm 2.3$ $\mathrm{U} / \mathrm{ml}$. While, there was no statically significant difference as regard relation of M2-PK to colorectal polyp risk however, it more elevated in high risk adenoma than non-adenomatous polyp. There was statically significant difference as regard relation of M2-PK to evidence of distant metastasis while there was no statically significant difference regarding sex, site, histological type, lymph node involvement and stage (table 4). Also, no statistical significant correlations between plasma M2 PK and age in CRC group ( $\mathrm{r}=0.06$; $\mathrm{P}=0.8$ ).

The performed analysis also focused on the determination of a cut-off for CRC prediction. This optimal cut-off was estimated at $>6 \mathrm{U} / \mathrm{mL}$ at AUC 0.949 with $83.33 \%$ sensitivity (missing rate was $16.67 \%$ ), $100 \%$ specificity, $100 \%$ PPV and 98.2\% NPV (table 3). These results support the role of plasma M2-PK in differentiating between CRC and normal individual. Also, plasma M2-PK can be used to discriminate between polyp group and CRC group at AUC 0.827 with a cut-off level of $>10.6 \mathrm{U} / \mathrm{ml}$, with $75.5 \%$ sensitivity, $87.5 \%$ specificity, $40.0 \%$ PPV and 96.9\% NPV (table 5). Also, our study showed that plasma M2-PK can be used to discriminate between to discriminate FBD from organic colonic lesion at AUC 0.872 with a cutoff level of $>3 \mathrm{U} / \mathrm{mL}$, with $81.94 \%$ sensitivity, $83.3 \%$ specificity, $35.3 \%$ PPV and $97.6 \%$ NPV (table 6). In our study we found positive correlation in direct proportion between plasma $\mathrm{M} 2-\mathrm{PK}$ and CEA $(\mathrm{r}=0.50 ; \mathrm{P}<0.001)$ and 
combination can give higher sensitivity and specificity.

Tables (1): Demographic features of the studied groups

\begin{tabular}{|c|c|c|c|c|c|c|c|}
\hline $\begin{array}{l}\text { Groups } \\
\text { Variables }\end{array}$ & & $\begin{array}{l}\text { Group I } \\
(n=24)\end{array}$ & $\begin{array}{l}\text { Group II } \\
(\mathbf{n}=\mathbf{2 4})\end{array}$ & $\begin{array}{l}\text { Group III } \\
(\mathrm{n}=\mathbf{2 4})\end{array}$ & $\begin{array}{l}\text { Group IV } \\
(\mathrm{n}=\mathbf{2 4})\end{array}$ & $\begin{array}{l}\text { Group V } \\
(\mathrm{n}=\mathbf{2 4})\end{array}$ & P-value \\
\hline \multirow{3}{*}{$\begin{array}{l}\text { Age } \\
\text { (years) }\end{array}$} & Mean & 44.83 & 41.88 & 34.54 & 45.08 & 50.38 & $<$ \\
\hline & \pm SD & 11.87 & 10.82 & 11.43 & 11.80 & 11.26 & $0.001 *$ \\
\hline & Range & $22-66$ & $20-59$ & $18-55$ & $18-65$ & $24-69$ & \\
\hline \multirow[t]{2}{*}{ Gender } & Male & $12(50 \%)$ & $\begin{array}{c}13 \\
(54.2 \%)\end{array}$ & $\begin{array}{c}10 \\
(41.7 \%)\end{array}$ & $\begin{array}{c}15 \\
(62.5 \%)\end{array}$ & $\begin{array}{c}14 \\
(58.3 \%)\end{array}$ & 0.6 \\
\hline & Female & $12(50 \%)$ & $\begin{array}{c}11 \\
(45.8 \%)\end{array}$ & $\begin{array}{c}14 \\
(58.3 \%)\end{array}$ & $9(37.5 \%)$ & $\begin{array}{c}10 \\
(41.7 \%)\end{array}$ & \\
\hline
\end{tabular}

*: p-value $<0.001$ is considered highly significant.

Table (2): Comparison between studied groups as regard ESR and CRP and tumor markers

\begin{tabular}{|c|c|c|c|c|c|c|c|}
\hline Variables & Groups & $\begin{array}{r}\text { Group I } \\
(\mathrm{n}=24)\end{array}$ & $\begin{array}{r}\text { Group II } \\
(\mathrm{n}=24)\end{array}$ & $\begin{array}{r}\text { Group } \\
\text { III } \\
(\mathrm{n}=24)\end{array}$ & $\begin{array}{r}\text { Group IV } \\
(n=24)\end{array}$ & $\begin{array}{r}\text { Group V } \\
(n=24)\end{array}$ & P-value \\
\hline \multirow{2}{*}{$\operatorname{ESR}(\mathbf{m m})$} & Mean & 6.83 & 8.04 & 12.38 & 17.75 & 29.21 & \\
\hline & \pm SD & 1.87 & 1.45 & 2.86 & 2.16 & 3.72 & $<0.001 *$ \\
\hline \multirow{3}{*}{ CRP (mg/dl) } & Mean & 3.38 & 3.71 & 8.29 & 4.54 & 5.00 & \\
\hline & \pm SD & 0.44 & 0.33 & 1.66 & 1.11 & 1.21 & $0.003 * *$ \\
\hline & Mean & 2.09 & 2.31 & 5.28 & 4.93 & 88.4 & \\
\hline CEA ng/mL & \pm SD & 0.72 & 0.03 & 1.33 & 1.61 & 17.96 & $<0.001 *$ \\
\hline M2-PK & Mean & 2.1 & 2.25 & 5.28 & 6.78 & 15.22 & \\
\hline $\mathrm{U} / \mathbf{m L}$ & \pm SD & 0.75 & 0.47 & 1.33 & 1.48 & 2.00 & $<0.001 *$ \\
\hline
\end{tabular}

*: p-value $<0.001$ is considered highly significant; **: p-value $<0.05$ is considered significant ESR: Erythrocyte sedimentation rate; CRP: C-reactive protein.

Table (3): Diagnostic performance of Plasma M2-PK and serum CEA in discrimination of normal group and CRC group

\begin{tabular}{llllllll}
\hline & Cut-off & $\begin{array}{r}\text { Area } \\
\text { under } \\
\text { the } \\
\text { curve }\end{array}$ & Sensitivity & Specificity & PPV & NPV & p-value \\
\hline M2-PK & $>6 \mathrm{U} / \mathrm{mL}$ & 0.949 & $83.33 \%$ & $100 \%$ & $100 \%$ & $98.2 \%$ & $<0.001^{*}$ \\
\hline CEA & $>7 \mathrm{ng} / \mathrm{mL}$ & 0.711 & $66.67 \%$ & $100 \%$ & $\begin{array}{l}100 \\
\%\end{array}$ & $96.4 \%$ & $0.0188^{* *}$ \\
\hline
\end{tabular}

*: p-value $<0.001$ is considered highly significant; $* *$ : p-value $<0.05$ is considered significant
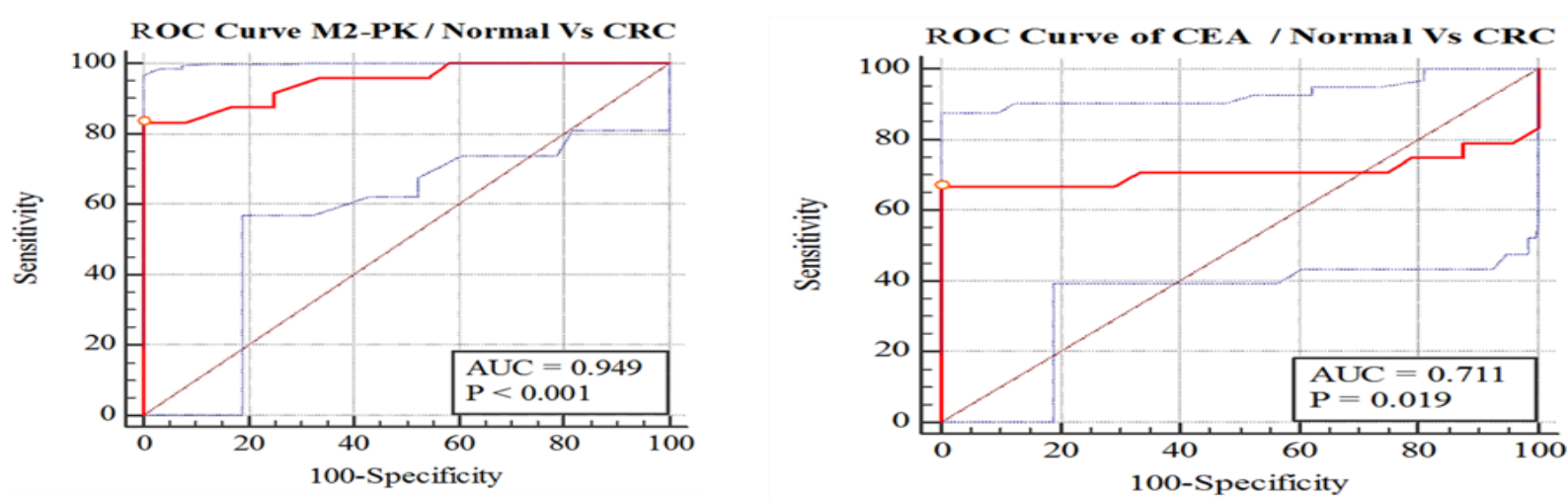
Figure (1): ROC curve between control group and CRC group as regard plasma M2-PK and serum CEA

Table (4): Relation of M2-PK to different prognostic factor in CRC patients

\begin{tabular}{|c|c|c|c|}
\hline Variant & & $\begin{array}{l}\text { M2-PK } \\
\text { Mean } \pm \text { SD }\end{array}$ & P-value \\
\hline Sex & Male (n=14) & $15.4 \pm 7.6$ & $\mathrm{P}=0.802$ \\
\hline & Female $(n=10)$ & $16.4 \pm 8.4$ & \\
\hline & Proximal $(n=8)$ & $12.8 \pm 7.85$ & \\
\hline Site of CRC & Distal (n=7) & $15.21 \pm 6.43$ & $P=0.522$ \\
\hline & $\operatorname{Rectum}(\mathbf{n}=9)$ & $17.37 \pm 9.39$ & \\
\hline Histopathological & Adenocarcinoma(n=21) & $14.54 \pm 7.92$ & $P=0.281$ \\
\hline type of CRC & $\begin{array}{l}\text { Mucinous } \\
\text { adenocarcinoma(n=3) }\end{array}$ & $19.97 \pm 8.36$ & \\
\hline Lymph & No $(n=10)$ & $12.13 \pm 6.15$ & $\mathrm{P}=0.112$ \\
\hline involvement & Yes $(n=14)$ & $17.42 \pm 8.63$ & \\
\hline Evidence of distant & No $(n=10)$ & $13.76 \pm 7.54$ & $* \mathrm{P}=0.043$ \\
\hline metastasis & Yes $(n=4)$ & $22.50 \pm 6.75$ & \\
\hline Stage & Stage I \& II (n=10) & $14.16 \pm 8.87$ & $P=0.596$ \\
\hline & Stage III \& IV $(n=14)$ & $15.97 \pm 7.57$ & \\
\hline
\end{tabular}

*: p-value $<0.05$ is considered significant

Table (5): Diagnostic performance of Plasma M2-PK and serum CEA in discrimination of polyp group and CRC group

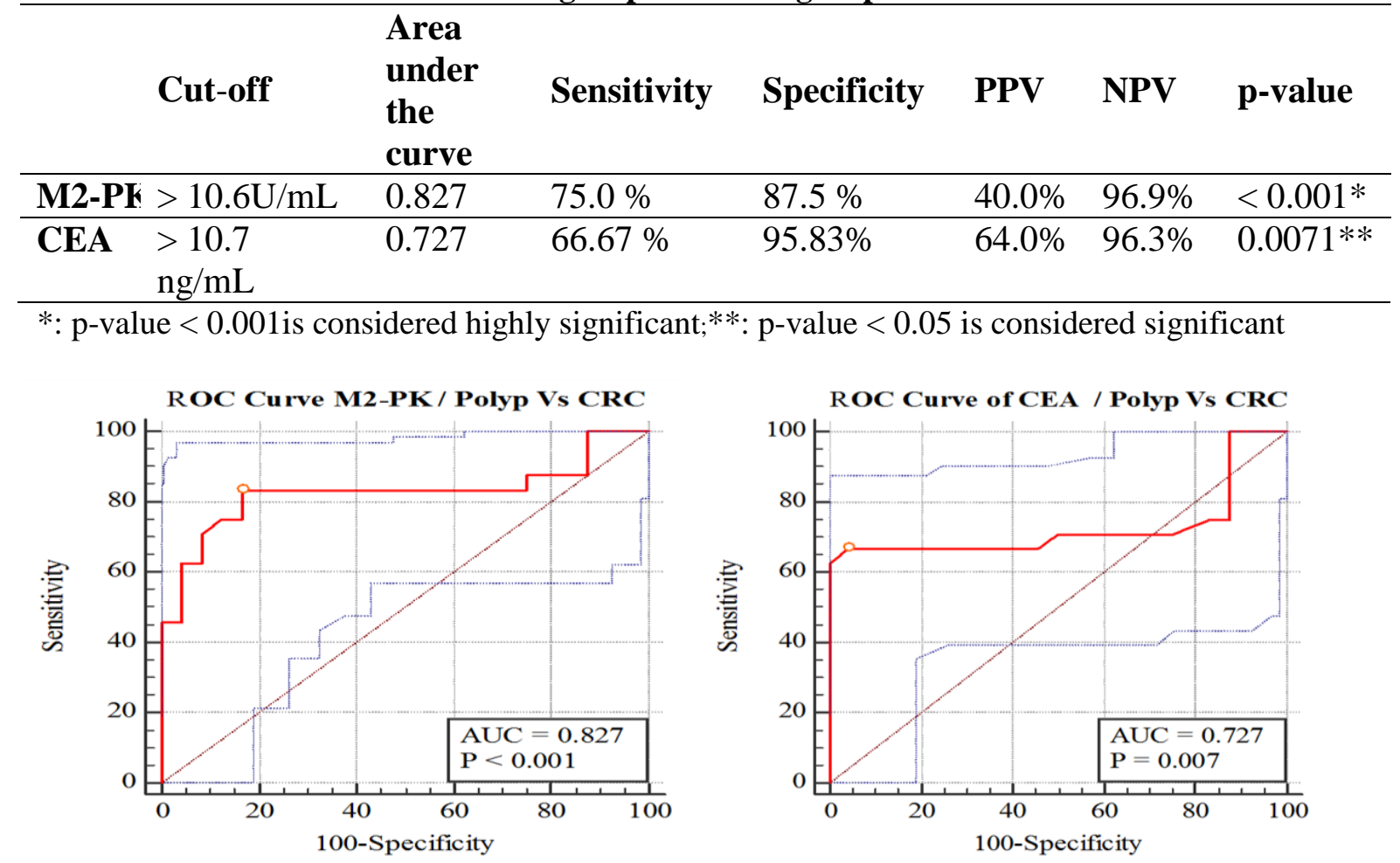

Figure (2): ROC curve between polyp group and CRC group as regard plasma M2-PK \& serum CEA 
Table (6): Diagnostic performance of Plasma M2-PK in discrimination of FBD from organic colonic lesions

\begin{tabular}{cllllll}
\hline \multicolumn{1}{c}{ Cut-off } & $\begin{array}{l}\text { Area } \\
\text { under } \\
\text { the } \\
\text { curve }\end{array}$ & Sensitivity & Specificity & PPV & NPV & p-value \\
\hline M2-PK $>3(\mathrm{U} / \mathrm{mL})$ & 0.872 & $81.94 \%$ & $83.33 \%$ & $35.3 \%$ & $97.6 \%$ & $<$ \\
& & & & & & $0.001 *$ \\
\hline
\end{tabular}

$*$ : p-value $<0.001$ is considered highly significant.

\section{Discussion}

Although, the recent advances in multidisciplinary management and treatment of $\mathrm{CRC}$, over the last years, no single test is currently able to diagnose. Patients are still reluctant to undergo screening tests because of the associated invasiveness and discomfort ${ }^{(\mathbf{1 6})}$. The ideal biomarker for CRC has not been found ${ }^{(17)}$. So, there is a critical need for reliable, minimally invasive, highly sensitive and specific markers of an individualized and optimized patient treatment at the earliest disease stage as possible ${ }^{(\mathbf{1 8})}$.

Our aim in the current study was to investigate the potential value of the plasma M2-PK in Egyptian patients with CRC and other colorectal lesions and compared its value to that of serum CEA to detect its sensitivity and specificity as a non-invasive biomarker in identification of such patients. We measured enzyme levels in EDTA-plasma samples as EDTA-plasma is the most appropriate sample for obtaining the diagnosis of this tumor marker (19). Tumor M2-PK can also be detected and measured in stool samples using an ELISA ${ }^{(20)}$. It was shown that fecal M2-PK is more accurate for CRC screening than plasma or serum tumor M2-PK ${ }^{(21)}$. However, blood tests are likely to be more acceptable than stool tests in population-based screening ${ }^{(22)}$.

The present study showed that there was highly statistical significant difference ( $p$-value $<0.001$ ) as regard plasma M2-PK, in CRC groups compared to other groups in all colorectal lesions. The mean plasma M2-PK level for CRC patients was $15.22 \pm 8 \mathrm{U} / \mathrm{ml}$. The plasma M2-PK can be used in differentiating between CRC and normal individual with $83.33 \%$ sensitivity, $100 \%$ specificity at (AUC) 0.949 with cut-off $>6 \mathrm{U} / \mathrm{ml}$ higher than that of CEA. Our results are in agreement with the study of Meng et $\boldsymbol{a l l}^{\left({ }^{(13)}\right.}$ which found significantly higher serum M2-PK in CRC patients compared to adenoma, nonadenomatous polyps, IBD and normal population with higher $94.62 \%$ sensitivity and low $55.06 \%$ specificity at cut-off $2.5 \mathrm{U} / \mathrm{ml}$. Hathurusinghe et $\boldsymbol{a l} .{ }^{(23)}$ reported in his a systematic review of an eight article that specificities were $89 \%$ or greater, sensitivity ranged from $47.8 \%$ to $76.5 \%$. Also, Bektafi $e t$ $\mathbf{a l} .^{(\mathbf{8})}$ in his study found that the CRC group had significantly higher levels of plasma M2-PK than the groups with adenomatous colorectal polyps and AUC was 0.664 the sensitivity was $35 \%$ and specificity was $99.33 \%$.Although, our performance is superior, but our study was carried on a small sample size $(n=24)$ beside the different best cut-off point and laboratory methods.

The current study showed that there was no statically significant difference as regard relation of M2-PK to age, sex, site, histopathological type, lymph node involvement and stage of CRC while, there was statically significant difference as regard relation of M2-PK to evidence of distant metastasis. Likely, Hathurusingh et $\boldsymbol{a l}^{(\mathbf{( 2 3 )}}$ found that patients with distant metastasis had higher levels of tumor M2-PK than patients without metastasis. Also, Fatela-Cantillo et al. ${ }^{(24)}$ supported this issue by finding that plasma M2-PK levels were significantly higher in patients with distant metastases and advanced stage than other which, indicated that plasma M2-PK testing on admission may have a potential prognostic role in identifying individuals with metastatic CRC and at an advanced stage. While, there was no relation to site of CRC and histopathological type of CRC. Also, Fatela-Cantillo et $\boldsymbol{a l l}^{\text {(24) }}$ suggested that plasmatic M2-PK may be a predictor of death risk when its plasma levels more than $20 \mathrm{U} / \mathrm{mL}$. 
In contrast, Kumaret al. ${ }^{(25)}$ could not find an association betweenM2-PK levels and advanced tumor stage and differentiation.

Munoz-Colmeneroet al. $^{(26)}$ in their study compared plasma tumor M2-PK levels in different cancer types. Nevertheless, various studies have demonstrated the increase of plasma M2-PK in different cancer (low specificity), the published literature is poor, only limited data exist on plasma tumor M2-PK in different cancer types such as breast, renal, lung, pancreatic and cervical cancer. We evaluated this marker in patient with colonic lesions and exclude any cancer other than CRC from the start and also we evaluate its relation to different prognostic factors and in differentiation of benign from malignant colonic lesions.

The current study showed that Plasma M2PK can be used to discriminate between benign from malignant colonic lesion at a cut-off level of $>10 \mathrm{U} / \mathrm{mL}$, with $83.33 \%$ sensitivity, $87.5 \%$ specificity, $42.6 \%$ PPV and $97.9 \%$ NPV.

Likely, Meng et al. ${ }^{(13)}$ and Bektafi et al. ${ }^{(8)}$ reported that plasma M2-PK can differentiate between benign and malignant colonic lesion. Unlikely, Fatela-Cantillo et $\boldsymbol{a l} .{ }^{(24)}$ stated that there is still no evidence that plasma M2-PK is sufficiently sensitive or specific to justify using it to differentiate malignant from benign colorectal disease for an individual patient.

The current study showed that plasma M2PK can be used to discriminate between to discriminate FBD from organic colonic lesion at a cut-off level of $>3 \mathrm{U} / \mathrm{mL}$, with $81.94 \%$ sensitivity, $83.3 \%$ specificity, $35.3 \%$ PPV and $97.6 \%$ NPV. Furthermore, the results of M2-PK levels in our study were shown to be significantly elevated in Myo endosopic score (1 \& 2), compared to normal or inactive UC.

Similarly, Bastawy et al. $^{(27)}$ revealed a highly significant increase in tumor M2-PK in the stool samples of patients with organic colonic disorders (IBD and CRC groups) compared to functional group (IBS). At a cutoff value of $4.2(\mathrm{U} / \mathrm{ml})$, the overall sensitivity and specificity for organic group over the functional group were $87.5 \%$ and $80 \%$ respectively. Additionally, the results of M2PK levels in his study were shown to be significantly elevated in active, compared to inactive IBD.

Data from Almousa et al. ${ }^{(28)}$ suggested that blood PKM2 as a promising biomarker for IBD and the dysbiosis of microflora in CD. Also,
Marin et al. ${ }^{(29)}$ showed that serum PKM2 levels appear to be a reliable biomarker of IBD activity. Likely, Chung-Faye $\boldsymbol{e t} \boldsymbol{a l} .^{(30)}$ in their study found high levels of M2-PK were documented in 81 adults diagnosed with IBD. This cohort was compared to a group of 43 subjects with IBS and 7 with CRC. M2-PK concentrations were higher in patients with IBD than in the controls. Furthermore, higher levels were evident in individuals with active IBD than in those with quiescent disease. In a further study by Jeffery $\boldsymbol{e t} \boldsymbol{a l} .{ }^{(\mathbf{3 1})} \mathrm{M} 2-\mathrm{PK}$ was assessed in 105 adults presenting with undifferentiated gastrointestinal symptoms and 94 healthy controls. The 14 adults subsequently diagnosed with organic diseases (only 10 with IBD) had higher fecal concentrations of M2-PK than those with functional symptoms or the controls. M2-PK measurement provided sensitivity of $67 \%$ and specificity of $88 \%$ in distinguishing between organic and functional diagnoses. However, it had a lower sensitivity, specificity and predictive value than fecal calprotectin in differentiation of organic disease from functional bowel disorders. However, this is in contradiction of the observation of Roszak $\boldsymbol{e t}$ $\boldsymbol{a l} .{ }^{(32)}$ who stated that M2-PK is a more sensitive marker than calprotectin and lactoferrin in evaluating disease activity in UC or CD.

In our study we find positive correlation in direct proportion between plasma M2-PK and CEA ( $\mathrm{r}=0.50 ; \mathrm{P}<0.001)$ and combination can give higher sensitivity and specificity.

In contrast, Meng et $\boldsymbol{a l} .{ }^{(13)}$ did not find a correlation between M2-PK and CEA levels $r=0.37, p=0.26)$. This may be due to the limited number of patients in his study as CEA levels measured in only 13 patients as well as due to a high standard deviation.

Dowling et $\boldsymbol{a l} .{ }^{(33)}$ in their study established that the level of several biomolecules, including M2P-K,serotonin, gamma enolase and members of the 14-3-3 family of proteins, showed statistically significant changes when comparing CRC patients with non-malignant patients. Also, Fung et al. ${ }^{(34)}$ identified a panel of three biomarkers (M2-PK, Insulin like growth factor binding protein 2 (IGFBP2), Dickkopf-, 3 and (DKK3) that discriminated between controls and CRC with $73 \%$ sensitivity at $95 \%$ specificity, or early stage (Stage I and II), raising the possibility for its use as a noninvasive blood diagnostic or screening test.

\section{Conclusion}


Plasma M2-PKlevels were significantly elevated in patients with CRC and may be helpful in distinguishing such patients from healthy subjects or patient with other colonic lesions. This suggests that the plasma M2-PK is fast non-invasive biomarker for CRC detection. Also, plasma M2-PK can differentiate between functional and organic colonic lesions.

\section{Recommendation}

Future researches including a large and long-term follow-up studies are recommended. Combined studies of different markers with plasma M2-PK either fecal or blood are recommended to increase the efficacy in diagnosis of CRC.

\section{References}

1. Mármol I, Sánchez-de-Diego C, Pradilla Dieste A, Cerrada E, Rodriguez Yoldi MJ (2017): Colorectal carcinoma: a general overview and future perspectives in colorectal cancer. International Journal of Molecular Sciences, 18(1): 197-236.

2. Fung KY, Tabor B, Buckley MJ, Priebe IK, Purins L, Pompeia Cet al. (2015): Bloodbased protein biomarker panel for the detection of colorectal cancer. PloS One, 10(3): e0120425.

3. Inadomi JM (2017): Screening for Colorectal Neoplasia. New England Journal of Medicine, 376(2):149-56.

4. Vatandoost $N$, Ghanbari J, Mojaver M, Avan A, Ghayour-Mobarhan M, Nedaeinia R et al. (2016): Early detection of colorectal cancer: from conventional methods to novel biomarkers. J Cancer Res Clin Oncology, 142(2): 341-51.

5. Zauber AG, Winawer SJ, O'brien MJ, Lansdorp-Vogelaar I, van Ballegooijen M, Hankey BF et al. (2012): Colonoscopic polypectomy and long-term prevention of colorectal-cancer deaths. New England Journal of Medicine, 366(8): 687-96.

6. Schoen RE, Pinsky PF, Weissfeld JL, Yokochi LA, Church T, Laiyemo AO et al. (2012):Colorectal-cancer incidence and mortality with screening flexible sigmoidoscopy. New England Journal of Medicine, 366(25): 2345-57.

7. Nikolouzakis TK, Vassilopoulou L, Fragkiadaki P, Mariolis Sapsakos T,
Papadakis GZ, Spandidos DA et al. (2018): Improving diagnosis, prognosis and prediction by using biomarkers in CRC patients (Review). Oncology Reports, 39(6): 2455-72.

8. Bektafi H, Remise G, fiGEN ÖT, ALTUNO E, LU HU, HUQ GE et al. (2013): Diagnostic and prognostic value of tumor M2pyruvate kinase levels in patients with colorectal cancer. Turkish J Gastroenterology, 24(1): 36-42.

9. Elwassief A, Soliman AI, Mostafa BE-DH, Matar ER (2014): Participation Rate and Results of Screening Colonoscopy in Egyptians with Family History of Colorectal Cancer. Nature and Science, 12(12): 29-34.

10. Costa F, Mumolo MG, Marchi S, Bellini M (2007): Differential diagnosis between functional and organic intestinal disorders: is there a role for non-invasive tests? World Journal of gastroenterology, 13(2): 219-23.

11. Conteduca V, Sansonno D, Russi S, Dammacco F (2013): Precancerous colorectal lesions. Internat J Oncology, 43(4): 973-84.

12. Huang J-X, Zhou Y, Wang C-H, Yuan W-W, Zhang Z-D, Zhang X-F (2014): Tumor M2-pyruvate kinase in stool as a biomarker for diagnosis of colorectal cancer: A meta-analysis. J Cancer Res Therapeutics, 10(7): 225-28.

13. Meng W, Zhu H-H, Xu Z-F, Cai S-R, Dong Q, Pan Q-R et al. (2012): Serum M2pyruvate kinase: a promising non-invasive biomarker for colorectal cancer mass screening. World J Gastrointestinal Oncology, 4(6): 14551.

14. Mazurek S (2012): Pyruvate kinase M2: A key enzyme of the tumor metabolome and its medical relevance. Biomedical Research (Aligarh), 23(2): SI 133-SI 41.

15. Li R, Liu J, Xue H, Huang G (2012): Diagnostic value of fecal tumor M2-pyruvate kinase for CRC screening: A systematic review and meta-analysis. Internat J Cancer, 131(8): 1837-45.

16. Pellino G, Gallo G, Pallante P, Capasso R, De Stefano A, Maretto I et al. (2018): Noninvasive Biomarkers of Colorectal Cancer: Role in Diagnosis and Personalised Treatment Perspectives. Gastroenterology Research and Practice, 2018(1): 1-21.

17. Álvarez-Chaver P, De Chiara L, Martínez Zorzano VS (2018): Proteomic Profiling for Colorectal Cancer Biomarker Discovery. Colorectal Cancer: Springer, 1765: 241-69. 
18. Das V, Kalita J, Pal M (2017): Predictive and prognostic biomarkers in colorectal cancer: A systematic review of recent advances and challenges. Biomedicine \& Pharmacotherapy, 87(3): 8-19.

19. Hugo F, Fischer G, Eigenbrodt E (1999): Quantitative detection of tumor M2-PK in serum and plasma. Anticancer Research, 19(4A): 2753-7.

20. Cho CH, Kim J, Jang MA, Lee BJ, Park J-J, Lim CS (2016): Evaluation of the performance of a fecal tumor M2-PK rapid kit using stool specimens for detection of colorectal tumors. Ann Clin Lab Scie., 46(2): $154-60$.

21. Tonus C, Sellinger M, Koss K, Neupert G (2012): Faecal pyruvate kinase isoenzyme type M2 for colorectal cancer screening: a metaanalysis. World J Gastroenterology, 18(30): 4004-11.

22. Adler A, Geiger S, Keil A, Bias H, Schatz $P$, Dhein J et al. (2014): Improving compliance to colorectal cancer screening using blood and stool based tests in patients refusing screening colonoscopy in Germany. BMC Gastroenterology, 14(1): 183-91.

23. Hathurusinghe HR, Goonetilleke KS, Siriwardena AK (2007): Current status of tumor M2 pyruvate kinase (tumor M2-PK) as a biomarker of gastrointestinal malignancy. Ann Surg Oncology, 14(10): 2714-20.

24. Fatela-Cantillo D, Fernandez-Suarez A, Moreno M, Gutierrez J, Iglesias J(2012): Prognostic value of plasmatic tumor M2 pyruvate kinase and carcinoembryonic antigen in the survival of colorectal cancer patients. Tumor Biology, 33(3): 825-32.

25. Kumar Y, Pinedo IR, Tapuria N, Zabron A, Davidson BR (2008): A comparison of tumour M2-PK with carcinoembryonic antigen and CA19-9 in patients undergoing liver resection for colorectal metastases. Euro $\mathrm{J}$ Gastroenterology Hepatology, 20(10): 100611.
26. Munoz-Colmenero A, Fernandez-Suarez A, Fatela-Cantillo D, Ocana-Perez E, Dominguez-Jimenez JL, Diaz-Iglesias JM (2015): Plasma tumor M2-Pyruvate kinase levels in different cancer types. Anticancer Research, 35(7): 4271-6.

27. Bastawy M, Elhawary M, Soliman $K$ (2017): Evaluation of Fecal Pyruvatekinase Isoenzyme (M2-Pk) Level in Differentiating Functional from Organic Colonic Disorders. Internat J Res Med Scie., 3(10): 112-9.

28. Almousa AA, Morris M, Fowler S, Jones J, Alcorn J (2018): Elevation of serum pyruvate kinase M2 (PKM2) in IBD and its relationship to IBD indices. Clinical Biochemistry, 53(4)19-24.

29. Marin R, Khalili H, Ananthakrishnan AN, Sauk J, Yajnik V, Gupta N et al. (2014): Sa1235 PKM2 As a Novel Serum Biomarker of IBD Activity: An Early Pilot Study. Gastroenterology, 146(5): S-238-S42.

30. Chung-Faye G, Hayee BH, Maestranzi S, Donaldson N, Forgacs I, Sherwood R (2007): Fecal M2-pyruvate kinase (M2-PK): a novel marker of intestinal inflammation. Inflammatory Bowel Diseases, 13(11): 1374-8. 31. Jeffery J, Lewis SJ, Ayling RM (2009): Fecal dimeric M2-pyruvate kinase (tumor M2PK) in the differential diagnosis of functional and organic bowel disorders. Inflammatory Bowel Diseases, 15(11): 1630-4.

32. Roszak D, Gałęcka M, Cichy W, Szachta $P$ (2015): Determination of faecal inflammatory marker concentration as a noninvasive method of evaluation of pathological activity in children with inflammatory bowel diseases. Adv Med Scie., 60(2): 246-52.

33. Dowling P, Hughes DJ, Larkin AM, Meiller J, Henry M, Meleady P et al. (2015): Elevated levels of 14-3-3 proteins, serotonin, gamma enolase and pyruvate kinase identified in clinical samples from patients diagnosed with colorectal cancer. Clin Chimica Acta, 441(1):133-41. 